\title{
A Formal Approach for Handling Lie Point Symmetries of Scalar First-Order Itô Stochastic Ordinary Differential Equations
}

\author{
Ebrahim FREDERICKS ${ }^{a}$ and Fazal M MAHOMED ${ }^{b}$ \\ a Mathematics and Applied Mathematics Bldg., Room 310.1, University of Cape Town, \\ Rondebosch, 7700, South Africa \\ E-mail: ebrahim.fredericks@uct.ac.za \\ ${ }^{b}$ School of Computational and Applied Mathematics, University of Witwatersrand, Wits \\ 2050, South Africa \\ E-mail: Fazal.Mahomed@wits.ac.za
}

\begin{abstract}
Many methods of deriving Lie point symmetries for Itô stochastic ordinary differential equations (SODEs) have surfaced. In the Itô calculus context both the formal and intuitive understanding of how to construct these symmetries has led to seemingly disparate results. The impact of Lie point symmetries on the stock market, population growth and weather SODE models, for example, will not be understood until these varying results are reconciled as has been attempted here.
\end{abstract}

\section{Introduction}

The adjustment of Lie's work for ordinary differential equations (ODEs) to Itô SODEs was first investigated by Gaeta and Quintero [4]; extended by Wafo Soh and Mahomed [10]; refined by Ünal [9] and reconciled by Fredericks and Mahomed [2]. The purpose of the work of Meleshko, Srihirun and Schultz [5] was to study a formal approach to finding the determining equations needed to obtain the Lie point infinitesimals. This paper seeks to correct them and reconcile [5] with the works of Wafo Soh and Mahomed [10], Ünal [9] and Fredericks and Mahomed [2].

Our first section introduces the transformation methology used in the Lie point approach. This is followed by an example done by Øksendal [7] to show how the random time change formula is used. How this is to be incorporated in the Lie point symmetry context is handled in the next section. Before proceding to do an example from [5] in the last section we construct the determining equations using a method similar to that of [5]. Unlike Meleshko, Srihirun and Schultz [5], we find the same determining equations which Fredericks and Mahomed [2] had found, which in turn reconciles [10] and [9]. 


\section{Transformations}

Books on SODEs by Revuz and Yor [8] and Freidlin [3] explain the nuances of random processes very well. One view of these processes is that they are a family of random variables indexed by a parameter, which in most instances is time. Thus for the spatial random process $X(t, \omega)$, each of its realisations $\omega$, are indexed as they progress through time. This paper deals entirely with Itô processes which are steered by Wiener processes. Wiener processes are stationary processes which have the following characteristics:

- at time zero with probability one, $W(0, \omega)=0$;

- for any strictly increasing sequence of indexed times $\left\{t_{i}\right\} \subset I$, the random variables $W\left(t_{i+1}, \omega\right)-W\left(t_{i}, \omega\right)$ are independent;

- for times $s<t, W(t, \omega)-W(s, \omega)$ is normally distributed with a zero mean and a variance of $t-s$.

- the covariance of a scalar process with itself at disparate times $\mathbb{E}(W(s, \omega) W(t, \omega))$ is just the minimum between the two different times $\min (t, s)$;

These characteristics give rise to Itô calculus, see, e.g., Brzeźniak and Zastawniak [1]. This calculus is able to find a SODE for which a function is a solution, if that function is itself at least once and twice differentialable w.r.t. time and space, respectively. Thus for an arbitrary function $F(t, x)$, which is at least once and twice differentialable w.r.t. time and space, Itô's calculus says that for a particular random spatial process $X(t, \omega)$ which satisfies

$$
d X(t, \omega)=f(X(t, \omega), \omega) d t+G(X(t, \omega), \omega) d W(t, \omega),
$$

$F(t, X(t, \omega))$ satisfies the SODE

$$
d F(t, X(t, \omega))=\left.\Gamma(F)\right|_{(t, X(t, \omega))} d t+\left.Y(F)\right|_{(t, X(t, \omega))} d W(t, \omega),
$$

where

$$
\begin{aligned}
\Gamma & =\frac{\partial}{\partial t}+f \frac{\partial}{\partial x}+\frac{1}{2} G^{2} \frac{\partial^{2}}{\partial x^{2}} \\
Y & =G \frac{\partial}{\partial x} .
\end{aligned}
$$

The necessary tools are now in place to carry out our Lie point symmetry analysis. As per Lie's work we begin with a one-parameter group of transformations of the time index and spatial random process, respectively

$$
\bar{t}=\theta(t, X(t, \omega), \epsilon) \quad \bar{X}(\bar{t}, \omega)=\varphi(t, X(t, \omega), \epsilon),
$$

where we link the group of transformations to the infinitesimal transformations $\bar{t} \approx t+\epsilon \tau$, $\bar{x} \approx x+\epsilon \xi$ via the following Lie relations

$$
\frac{\partial \theta}{\partial \epsilon}=\tau(\theta, \varphi) \quad \frac{\partial \varphi}{\partial \epsilon}=\xi(\theta, \varphi)
$$


which have the initial conditions at $\epsilon=0$, i.e.

$$
\left.\bar{t}\right|_{\epsilon=0}=t, \quad \text { and }\left.\bar{X}(\bar{t}, \omega)\right|_{\epsilon=0}=X(t, \omega) .
$$

Whence the differential infinitesimal transformations are

$$
d \bar{t}=d t+\epsilon d \tau(\theta, \varphi)(t, X(t, \omega))+\mathcal{O}\left(\epsilon^{2}\right)
$$

and

$$
d \bar{X}(\bar{t}, \omega)=d X(t, \omega)+\epsilon d \xi(\theta, \varphi)(t, X(t, \omega))+\mathcal{O}\left(\epsilon^{2}\right) .
$$

\section{An Example of Random Time Change from [7]}

Since random processes which can be viewed as families of random variables indexed by time are non-deterministic, we use the random time change formula from Øksendal [6] to perform a random time change $\bar{t}=\beta(t, \omega)$, where we introduce the measureable time change rate $c(t, \omega)$ which is related to our time change scalar stochastic process $\beta(t, \omega)$ by the equation

$$
\beta(t, \omega)=\int^{t} c(s, \omega) d s
$$

and $\alpha(t, \omega)$ be a scalar stochastic process satisfying

- $\alpha(0, \omega)=0$,

- $d \alpha(t, \omega) / d t=1 / c(\alpha(t, \omega), \omega) \geq 0$, for almost all positive time and almost all $\omega \in \Omega$.

- $\beta(t, \omega)$ and $\alpha(t, \omega)$ are left and right inverses of each other respectively, $\alpha(\beta(t, \omega), \omega)=\beta(\alpha(t, \omega), \omega)=t$ for all $(t, \omega) \in I \times \Omega$.

We illustrate the use of the random time change formula via an example in Øksendal [7],

$$
d Y(t, \omega)=\frac{1}{|B(t, \omega)|} \sigma(Y(t, \omega)) d B(t, \omega)+\frac{1}{|B(t, \omega)|^{2}} b(Y(t, \omega)) d t
$$

Now perform the following time change: define

$$
Z(t, \omega)=Y(\alpha(t, \omega), \omega)
$$

Thus we have that 


$$
\begin{array}{r}
d Y(\alpha(t, \omega), \omega)=\frac{1}{|B(\alpha(t, \omega), \omega)|} \sigma(Y(\alpha(t, \omega), \omega)) d B(\alpha(t, \omega), \omega) \\
\quad+\frac{1}{|B(\alpha(t, \omega), \omega)|^{2}} b(Y(\alpha(t, \omega), \omega)) d \alpha(t, \omega)
\end{array}
$$

where

$$
\beta(t, \omega)=\int_{0}^{t} c(s, \omega) d s \text { and } c(t, \omega)=\frac{1}{|B|^{2}}
$$

which means that

$$
d \tilde{B}(t, \omega)=\frac{1}{|B(\alpha(t, \omega), \omega)|} d B(\alpha(t, \omega), \omega)
$$

and

$$
d t=\frac{d \alpha(t, \omega)}{|B(\alpha(t, \omega), \omega)|^{2}}
$$

whence

$$
d Z(t, \omega)=\sigma(Z(t, \omega)) d \tilde{B}(t, \omega)+b(Z(t, \omega)) d t
$$

when using the random time change formula for Itô integrals.

\section{Using Random Time Change for Lie Symmetry Methods}

Continuing from the previous section we have the following relation between the rate of time change and time change itself,

$$
\bar{t}=\int_{0}^{t} c(s, \omega) d s=t+\epsilon \tau(t, X(t, \omega))+\mathcal{O}\left(\epsilon^{2}\right) .
$$

We then use Itô's formula to get the following relation

$$
\int_{0}^{t} c(s, \omega) d s=\int_{0}^{t}\left(1+\left.\epsilon \Gamma(\tau)\right|_{(t, X(t, \omega))}\right) d s+\left.\epsilon \int_{0}^{t} Y(\tau)\right|_{(t, X(t, \omega))} d W(s)+\mathcal{O}\left(\epsilon^{2}\right),
$$

where $\Gamma$ is as in (2.3) and $Y$ is defined in (2.4). These are derived from the spatial Itô process

$$
d X(t, \omega)=f(t, X(t, \omega)) d t+G(t, X(t, \omega)) d W(t)
$$


which implies that $Y(\tau(t, X(t, \omega)))=0$, if we compare Riemann and Itô integrals. This temporal infinitesimal condition concurrs with that of [9]. We use the random time change formula for Itô integrals

$$
\int_{0}^{t} d \bar{W}(s)=\int_{0}^{\alpha(t, \omega)} \sqrt{c(s, \omega)} d W(s)
$$

which can also be used to give the following relation

$$
\int_{0}^{\bar{t}} d \bar{W}(s)=\int_{0}^{t} \sqrt{c(s, \omega)} d W(s)
$$

since $\alpha(t, \omega)$ is the left-inverse of $\beta(t, \omega)$.

\section{Correction of the work [5]}

In the paper [5] the following relation was made among $\tau(t, X(t, \omega)), c(t, \omega)$ and the new variable $\eta(t, \omega)$

$$
\begin{aligned}
\eta(t, \omega, \epsilon) & =\sqrt{c(t, \omega)} \\
\left.\frac{\partial \eta(t, \omega, \epsilon)}{\partial \epsilon}\right|_{\epsilon=0} & =\frac{1}{2} \frac{d \tau(t, X(t, \omega))}{d t}
\end{aligned}
$$

since $c(t, \omega)=1$ at $\epsilon=0$. This is NOT a miss printed relation from [5]; it has just been rewritten to be consistent with the notation [2] used in their paper. It was further stated in [5] that $d \tau(t, X(t, \omega)) /(2 d t)$ and the spatial infinitesimal $\xi(t, \omega)$ defines a Lie group transformation for stochastic processes. From here onwards we wish to correct [5] in finding the determining equations.

Using Itô's formula on $\theta(t, X(t, \omega), \epsilon)$ and $\varphi(t, X(t, \omega), \epsilon)$ gives

$$
\begin{aligned}
\theta(t, X(t, \omega), \epsilon)=\theta & (0, X(0, \omega), \epsilon)+\left.\int_{0}^{t} \Gamma(\theta)\right|_{(s, X(s, \omega), \epsilon)} d s \\
& +\left.\int_{0}^{t} Y(\theta)\right|_{(s, X(s, \omega), \epsilon)} d W(s)
\end{aligned}
$$

and

$$
\begin{aligned}
\varphi(t, X(t, \omega), \epsilon)=\varphi & (0, X(0, \omega), \epsilon)+\left.\int_{0}^{t} \Gamma(\varphi)\right|_{(s, X(s, \omega), \epsilon)} d s \\
& +\left.\int_{0}^{t} Y(\varphi)\right|_{(s, X(s, \omega), \epsilon)} d W(s) .
\end{aligned}
$$


Form invariance arguments on the integrands require

$$
\begin{array}{r}
\bar{X}(\beta(t, \omega), \omega)=\bar{X}(0, \omega)+\int_{0}^{t} f(\beta(s, \omega), \bar{X}(\beta(s, \omega), \omega)) d \beta(s, \omega) \\
+\int_{0}^{t} G(\beta(s, \omega), \bar{X}(\beta(s, \omega), \omega)) d \bar{W}(\beta(s, \omega))
\end{array}
$$

which is totally different to what [5] had, where the form invariance argument was only applied to the interval of integration and not to the time indices which followed the transformed spatial random process along the time interval. This can also be represented as

$$
\begin{aligned}
\bar{X}(\beta(t, \omega), \omega)=\bar{X}(0, \omega)+\int_{0}^{t} f(\beta(s, \omega), \bar{X}(\beta(s, \omega), \omega)) c(s, \omega) d s \\
+\int_{0}^{t} G(\beta(s, \omega), \bar{X}(\beta(s, \omega), \omega)) \sqrt{c(s, \omega)} d W(s),
\end{aligned}
$$

where we used the relation $d \beta(s, \omega) / d s=c(s, \omega)$ and $d W(\beta(s, \omega)) / d W(s)=\sqrt{c(s, \omega)}$ from (4.1) and (4.5) respectively. This means that we must differentiate (5.5) and (5.4) by $\epsilon$ at $\epsilon=0$ and then equate their results in order to establish the determining equations. Differentiating (5.4) and (5.5) by $\epsilon$ gives

$$
\begin{aligned}
\frac{\partial \varphi}{\partial \epsilon}=\frac{\partial}{\partial \epsilon} & \left(\varphi(0, X(0, \omega), \epsilon)+\left.\int_{0}^{t} \Gamma(\varphi)\right|_{(s, X(s, \omega), \epsilon)} d s\right. \\
& \left.+\left.\int_{0}^{t} Y(\varphi)\right|_{(s, X(s, \omega), \epsilon)} d W(s)\right) \\
=\xi & (0, X(0, \omega))+\mathcal{O}(\epsilon)+\left.\int_{0}^{t} \Gamma(\xi+\mathcal{O}(\epsilon))\right|_{(s, X(s, \omega))} d s \\
& +\left.\int_{0}^{t} Y(\xi+\mathcal{O}(\epsilon))\right|_{(s, X(s, \omega), \epsilon)} d W(s)
\end{aligned}
$$


and

$$
\begin{aligned}
\frac{\partial \bar{X}(\beta(t, \omega), \omega)}{\partial \epsilon} & =\frac{\partial \bar{X}(0, \omega)}{\partial \epsilon}+\int_{0}^{t}\left(\frac{\partial f(\beta(s, \omega), \bar{X}(\beta(s, \omega), \omega))}{\partial \bar{t}} \frac{\partial \beta(s, \omega)}{\partial \epsilon}\right. \\
& +\frac{\partial f(\beta(s, \omega), \bar{X}(\beta(s, \omega), \omega))}{\partial \bar{x}} \frac{\partial \bar{X}(\beta(s, \omega), \omega)}{\partial \epsilon} \\
& \left.+f(\beta(s, \omega), \bar{X}(\beta(s, \omega), \omega)) \frac{\partial}{\partial \epsilon}(c(s, \omega))\right) d s \\
& +\int_{0}^{t}\left(\frac{\partial G(\beta(s, \omega), \bar{X}(\beta(s, \omega), \omega))}{\partial \bar{t}} \frac{\partial \beta(s, \omega)}{\partial \epsilon}\right. \\
& +\frac{\partial G(\beta(s, \omega), \bar{X}(\beta(s, \omega), \omega))}{\partial \bar{x}} \frac{\partial \bar{X}(\beta(s, \omega), \omega)}{\partial \epsilon} \\
& \left.+G(\beta(s, \omega), \bar{X}(\beta(s, \omega), \omega)) \frac{\partial}{\partial \epsilon}(\sqrt{c(s, \omega)})\right) d W(s, \omega) .
\end{aligned}
$$

Therefore at $\epsilon=0$ we arrive at

$$
\begin{aligned}
\xi(t, X(t, \omega))=\xi( & 0, X(0, \omega))+\left.\int_{0}^{t} \Gamma(\xi)\right|_{(s, X(s, \omega))} d s \\
& +\left.\int_{0}^{t} Y(\xi)\right|_{(s, X(s, \omega))} d W(s, \omega)
\end{aligned}
$$

and

$$
\begin{aligned}
\left.\frac{\partial \bar{X}(\alpha(t, \omega), \omega)}{\partial \epsilon}\right|_{\epsilon=0} & =\xi(\theta, \varphi)(0, X(0, \omega)) \\
& +\left.\int_{0}^{t}\left(\frac{\partial f}{\partial s} \tau(\theta, \varphi)+\frac{\partial f}{\partial x} \xi(\theta, \varphi)+f \Gamma(\tau)(\theta, \varphi)\right)\right|_{(s, X(s, \omega))} d s \\
& +\left.\int_{0}^{t}\left(\frac{\partial G}{\partial s} \tau(\theta, \varphi)+\frac{\partial G}{\partial x} \xi(\theta, \varphi)+\frac{1}{2} G \Gamma(\tau)(\theta, \varphi)\right)\right|_{(s, X(s, \omega))} d W(s) .
\end{aligned}
$$

If we now compare the Rieman and Itô integrands we find the same determining equations that [2] found, viz.

$$
\begin{aligned}
\Gamma(\xi) & =H(f)+f \Gamma(\tau), \\
Y(\xi) & =H(G)+\frac{1}{2} G \Gamma(\tau)
\end{aligned}
$$

and the extra condition

$$
Y(\tau)=0,
$$


where

$$
H=\tau \frac{\partial}{\partial t}+\xi \frac{\partial}{\partial x}
$$

and $\Gamma$ and $Y$ are given by (2.3) and (2.4).

We re-consider two examples from [5] followed by a more general problem which was not done in $[5]$.

\section{$5.1 \quad$ Example 1}

Consider

$$
d X(t, \omega)=\mu X(t, \omega) d t+\sigma X(t, \omega) d W(t, \omega)
$$

with the initital condition $X(0, \omega)=x_{0}$. The determining equations are

$$
\Gamma(\xi(t, x))=H(\mu x)+\mu x \Gamma(\tau(t)),
$$

or equivalently

$$
\frac{\partial \xi(t, x)}{\partial t}+\mu x \frac{\partial \xi(t, x)}{\partial x}+\frac{\sigma^{2} x^{2}}{2} \frac{\partial^{2} \xi(t, x)}{\partial x^{2}}=\xi(t, x) \mu+\mu x \frac{d \tau(t)}{d t}
$$

and

$$
Y(\xi(t, x))=\xi(t, x) \sigma+\frac{\sigma x}{2} \frac{d \tau(t)}{d t}
$$

which is

$$
x \frac{\partial \xi(t, x)}{\partial x}=\xi(t, x)+\frac{x}{2} \frac{d \tau(t)}{d t}
$$

since the extra condition forces the temporal infinitesimal, $\tau$, to be a function of time only. By substituting for $\xi(t, x)$ from (5.14), (5.13) becomes

$$
\frac{\partial \xi(t, x)}{\partial t}+\frac{\sigma^{2} x^{2}}{2} \frac{\partial^{2} \xi(t, x)}{\partial x^{2}}=\frac{\mu x}{2} \frac{d \tau(t)}{d t} .
$$

Multiplying (5.14) by $x$ and then differentiating by $x$ gives

$$
\begin{aligned}
x^{2} \frac{\partial^{2} \xi(t, x)}{\partial x^{2}} & =-x \frac{\partial \xi(t, x)}{\partial x}+\xi(t, x)+x \frac{d \tau(t)}{d t} \\
& =\frac{x}{2} \frac{d \tau(t)}{d t}
\end{aligned}
$$


after using the result (5.14) again. Thus (5.15) becomes

$$
\frac{\partial \xi(t, x)}{\partial t}=\frac{1}{2}\left(\mu-\frac{1}{2} \sigma^{2}\right) x \frac{d \tau(t)}{d t}
$$

which means that

$$
\xi(t, x)=\frac{1}{2}\left(\mu-\frac{1}{2} \sigma^{2}\right) x \tau(t)+a_{1}(x),
$$

where $a_{1}(x)$ is an arbitrary function of $x$. Equation (5.16) now becomes

$$
a_{1}^{\prime \prime}(x)=\frac{\dot{\tau}(t)}{2 x}
$$

which in turn yields

$$
a_{1}(x)=\frac{1}{2} \dot{\tau}(t)(x \ln x-x)+a_{2} x+a_{3},
$$

where $a_{2}$ and $a_{3}$ are arbitrary constants. By substitution of (5.18) with $a_{1}$ in (5.20) into (5.13), we have that the temporal infinitesimal reduces to

$$
\tau(t)=a_{4}+a_{5} t
$$

and the spatial infinitesimal becomes

$$
\xi(t, x)=\frac{1}{2}\left(\mu-\frac{1}{2} \sigma^{2}\right) x\left(a_{4}+a_{5} t\right)+a_{2} x+\frac{1}{2} a_{5}(x \ln x-x) .
$$

We demonstrate that the finite transformations are recoverable by considering the nontrivial case, i.e. $a_{4}=1$ and $a_{i}=0$ for $i=2$ and 5

$$
\begin{aligned}
\frac{d \bar{x}}{d \epsilon} & =\frac{1}{2}\left(\mu-\frac{1}{2} \sigma^{2}\right) \bar{x} \\
\frac{d \bar{t}}{d \epsilon} & =1 .
\end{aligned}
$$

Solving (5.24) gives

$$
\bar{t}=t .
$$

Solving for $\bar{x}$ leaves us with the following

$$
\bar{x}=e^{\frac{1}{2}\left(\mu-\frac{1}{2} \sigma^{2}\right) \epsilon} x .
$$

The Itô formula trivially gives the form invariant SDE 


$$
\begin{aligned}
& d \bar{X}(t, \omega)=\mu X(t, \omega) e^{\frac{1}{2}\left(\mu-\frac{1}{2} \sigma^{2}\right) \epsilon} d t+\sigma X(t, \omega) e^{\frac{1}{2}\left(\mu-\frac{1}{2} \sigma^{2}\right) \epsilon} d W(t, \omega) \\
& \quad=\mu \bar{X}(\bar{t}, \omega) d \bar{t}+\sigma \bar{X}(\bar{t}, \omega) d \bar{W}(\bar{t}, \omega)
\end{aligned}
$$

which makes sense, since there is no random time change for this example. However, in [5], the symmetries found do not maintain the properties of the transformed Wiener processes. In trying to show that the finite transformations are retrievable from the infinitesimal ones, the work of [5] uses the spatial and temporal infinitesimal values directly up to order $\mathcal{O}(\epsilon)$ and falsely claim that the higher order infinitesimals will agree with the Itô formula applied to the finite group transformations, which are found using the Lie equations.

Focusing on the non-trivial finite group of transformations which was found in [5], i.e.

$$
\bar{x}=\left(\epsilon+x^{-\gamma}\right)^{-\frac{1}{\gamma}}
$$

and

$$
\bar{t}=t\left(1+\epsilon x^{\gamma}\right)^{-2}
$$

where $\gamma=\frac{2 \mu}{\sigma^{2}}-1$, which we are able to derive using the spatial and temporal infinitesimals,

$$
\tau=2 t x^{\gamma}
$$

and

$$
\xi=\frac{x^{\gamma-1}}{\gamma}
$$

respectively, we see that the invariant condition is not satisfied. We have that the Itô formula gives the following for the transformed spatial process

$$
\begin{aligned}
d \bar{X}(\bar{t}, \omega)= & \Gamma(\bar{x})(t, \omega) d t+Y(\bar{x})(t, \omega) d W(t, \omega) \\
= & \left(\epsilon+X^{-\gamma}\right)^{-\frac{1}{\gamma}-2} X^{-\gamma}\left(\mu\left(\epsilon+X^{-\gamma}\right)-\frac{\epsilon \sigma^{2}}{2}(1+\gamma)\right) d t \\
& +\sigma\left(\epsilon+X^{-\gamma}\right)^{-\frac{1}{\gamma}-1} X^{-\gamma} d W(t, \omega),
\end{aligned}
$$

while for the transformed time index, we have

$$
\begin{aligned}
d \bar{t}(\bar{t}, \omega)= & \Gamma(\bar{t})(t, \omega) d t+Y(\bar{t})(t, \omega) d W(t, \omega) \\
= & \left(1+\epsilon X^{\gamma}\right)^{-2}\left(1-2 t \gamma \mu \epsilon X^{\gamma}\left(1+\epsilon X^{\gamma}\right)^{-1}\right. \\
& \left.-\frac{t \sigma^{2}}{2}\left(2 \gamma(\gamma-1) \epsilon X^{\gamma}\left(1+\epsilon X^{\gamma}\right)^{-1}-6 \gamma^{2} \epsilon^{2} X^{2 \gamma}\left(1+\epsilon X^{\gamma}\right)^{-2}\right)\right) d t \\
& -2 \sigma t \gamma \epsilon X^{\gamma}\left(1+\epsilon X^{\gamma}\right)^{-3} d W(t, \omega) .
\end{aligned}
$$


The Itô formula for the transformed time index can never be recovered in that of the transformed spatial processes because the variable $t$ appears nowhere in (5.33). We continue with a brief study of the relations between the three generators of symmetry which we have found, viz.

$$
\begin{aligned}
& H_{1}=\frac{\partial}{\partial t}+\frac{1}{2}\left(\mu-\frac{1}{2} \sigma^{2}\right) x \frac{\partial}{\partial x} \\
& H_{2}=x \frac{\partial}{\partial x} \\
& H_{3}=t \frac{\partial}{\partial t}+\frac{1}{2}\left(\left(\mu-\frac{1}{2} \sigma^{2}\right) t x+x \ln x-x\right) \frac{\partial}{\partial x} .
\end{aligned}
$$

The Lie bracket relations are

$$
\left[H_{1}, H_{2}\right]=0,\left[H_{1}, H_{3}\right]=H_{1}+\frac{1}{4}\left(\mu-\frac{1}{2} \sigma^{2}\right) H_{2},\left[H_{2}, H_{3}\right]=\frac{1}{2} H_{2} .
$$

Therefore we have a three-dimensional algebra of symmetry generators.

\subsection{Example 2}

Now we have

$$
d X(t, \omega)=\mu d t+d W(t, \omega)
$$

with the initital condition $X(0, \omega)=x_{0}$. The determining equations are

$$
\Gamma(\xi(t, x))=H(\mu)+\mu \Gamma(\tau(t))
$$

or

$$
\frac{\partial \xi(t, x)}{\partial t}+\mu \frac{\partial \xi(t, x)}{\partial x}+\frac{\partial^{2} \xi(t, x)}{2 \partial x^{2}}=\mu \frac{d \tau(t)}{d t}
$$

and

$$
Y(\xi(t, x))=\frac{1}{2} \Gamma(\tau(t))
$$

which in conjunction with Ünal's [9] extra condition forces the following relation between the spatial and temporal infinitesimals

$$
\frac{\partial \xi(t, x)}{\partial x}=\frac{1}{2} \dot{\tau}(t)
$$

Substituting $\xi(t, x)$ from (5.40) into (5.39), causes the equation (5.39) to become

$$
\frac{\partial \xi(t, x)}{\partial t}=\frac{1}{2} \mu \dot{\tau}(t)
$$


which due to the compatibility of (5.40) and (5.41) results in

$$
\tau(t, x)=a_{1}+a_{2} t
$$

where $a_{1}$ and $a_{2}$ are arbitrary constants. Then (5.40) and (5.41) imply

$$
\xi=\frac{1}{2} a_{2} x+\frac{1}{2} \mu a_{2} t+a_{3}
$$

where $a_{3}$ is an arbitrary constant. The finite group of transformations for $a_{2}=2$ and rest zero are solved from the following equations

$$
\frac{d \bar{x}}{d \epsilon}=\mu \bar{t}+\bar{x},\left.\quad \bar{x}\right|_{\epsilon=0}=x
$$

and

$$
\frac{d \bar{t}}{d \epsilon}=2 \bar{t},\left.\quad \bar{t}\right|_{\epsilon=0}=t
$$

The temporal group of transformations is easily solved to give

$$
\bar{t}=t e^{2 \epsilon},
$$

which induces the following ODE from (5.44)

$$
\frac{d \bar{x}}{d \epsilon}-\bar{x}=\mu t e^{2 \epsilon} .
$$

Solving gives

$$
\bar{x}=(x-\mu t) e^{\epsilon}+\mu t e^{2 \epsilon} .
$$

Applying Itô's formula to (5.48) yields

$$
\begin{aligned}
d \bar{X}(\bar{t}, \omega) & =(\mu d t+d W(t, \omega)-\mu d t) e^{\epsilon}+\mu d t e^{2 \epsilon} \\
& =\mu e^{2 \epsilon} d t+e^{\epsilon} d W(t, \omega) \\
& =\mu d \bar{t}+d \bar{W}(\bar{t}, \omega) .
\end{aligned}
$$

Thus form invariance is maintained. This is not true for the following transformations which were found in [5]:

$$
\bar{x}=x-\frac{1}{2 \mu} \ln \left(1-2 \mu \epsilon e^{2 \mu x}\right)
$$

and

$$
\bar{t}=t\left(1-2 \mu \epsilon e^{2 \mu x}\right)^{-2} .
$$


The Itô formula for the spatial transformations is

$$
\begin{aligned}
d \bar{X}(\bar{t}, \omega)= & \left(\mu\left(1+\frac{4 \mu^{2} \epsilon e^{2 \mu x}}{2 \mu\left(1-2 \mu \epsilon e^{2 \mu x}\right)}\right)\right. \\
& \left.+\frac{8 \mu^{3} \epsilon e^{2 \mu x}}{4 \mu\left(1-2 \mu \epsilon e^{2 \mu x}\right)^{2}}\right) d t \\
& +\left(1+\frac{4 \mu^{2} \epsilon e^{2 \mu x}}{2 \mu\left(1-2 \mu \epsilon e^{2 \mu x}\right)}\right) d W(t, \omega) .
\end{aligned}
$$

As with the previous example there is no presence of a time index in the drift or diffusion coefficients. Calculating the Itô formula for the transformed time index gives

$$
\begin{aligned}
d \bar{t}(\bar{t}, \omega)= & \left(\frac{1}{\left(1-2 \mu \epsilon e^{2 \mu x}\right)^{2}}+t \mu\left(\frac{8 \mu^{2} \epsilon e^{2 \mu x}}{\left(1-2 \mu \epsilon e^{2 \mu x}\right)^{3}}\right)\right. \\
& \left.+\frac{8 t \mu^{3} \epsilon e^{2 \mu x}}{\left(1-2 \mu \epsilon e^{2 \mu x}\right)^{3}}+\frac{48 t \mu^{4} \epsilon^{2} e^{4 \mu x}}{\left(1-2 \mu \epsilon e^{2 \mu x}\right)^{4}}\right) d t \\
& +\frac{8 \mu^{2} \epsilon t e^{2 \mu x}}{\left(1-2 \mu \epsilon e^{2 \mu x}\right)^{3}} d W(t, \omega) .
\end{aligned}
$$

The consequence of failing to comply with condition (5.11), leads to symmetries which do not leave the transformed spatial process form invariant. Though [5] demonstrates that the transformed Wiener process obeys the random time change formula, form invariance for the transformed spatial process is deficient.

The symmetry generators we obtain are

$$
\begin{aligned}
& H_{1}=\frac{\partial}{\partial t} \\
& H_{2}=\frac{\partial}{\partial x} \\
& H_{3}=2 t \frac{\partial}{\partial t}+(\mu t+x) \frac{\partial}{\partial x}
\end{aligned}
$$

which have the following Lie bracket relations

$$
\left[H_{1}, H_{2}\right]=0,\left[H_{1}, H_{3}\right]=2 H_{1}+\mu H_{2},\left[H_{2}, H_{3}\right]=H_{2} .
$$

\subsection{Example 3}

We finally investigate

$$
d X(t, \omega)=f(t) d t+g(t) d W(t, \omega)
$$

with the initital condition $X(0, \omega)=x_{0}$. Here the determining equations are

$$
\Gamma(\xi(t, x))=H(f(t))+f(t) \Gamma(\tau(t)),
$$


which gives

$$
\frac{\partial \xi(t, x)}{\partial t}+f(t) \frac{\partial \xi(t, x)}{\partial x}+\frac{g^{2}(t)}{2} \frac{\partial^{2} \xi(t, x)}{\partial x^{2}}=\tau(t) \frac{\partial f(t)}{\partial t}+f(t) \frac{d \tau(t)}{d t}
$$

and

$$
Y(\xi(t, x))=H(g(t))+\frac{g(t)}{2} \Gamma(\tau(t, x))
$$

Applying Ünal's [9] extra condition forces the following relation between the spatial and temporal infinitesimals

$$
\frac{\partial \xi(t, x)}{\partial x}=\tau(t) \frac{\partial \ln g(t)}{\partial t}+\frac{1}{2} \dot{\tau}(t)
$$

Substituting $\xi(t, x)$ from (5.58) into (5.57) results in the equation (5.57) becoming

$$
\frac{\partial \xi(t, x)}{\partial t}+f(t) \tau(t) \frac{\partial \ln g(t)}{\partial t}=\tau(t) \frac{\partial f(t)}{\partial t}+f(t) \frac{1}{2} \dot{\tau}(t),
$$

Integrating (5.58) w.r.t. the spatial coordinate $x$ and then using the time differentiated result of this calculation to perform a comparison within (5.59) via separation of coefficients w.r.t. the spatial variable $x$, leads to the following

$$
\tau(t) \frac{\partial \ln g(t)}{\partial t}+\frac{1}{2} \dot{\tau}(t)=C_{0}
$$

and

$$
\dot{m}(t)=-f(t) \tau(t) \frac{\partial \ln g(t)}{\partial t}+\tau(t) \frac{\partial f(t)}{\partial t}+f(t) \frac{1}{2} \dot{\tau}(t)
$$

where $C_{0}$ and $m(t)$ are an arbitrary constant and arbitrary function of time respectively, which constitute the spatial infinitesimal as

$$
\xi(t, x)=C_{0} x+m(t)
$$

Solving for the temporal infinitesimal from (5.60) gives

$$
\tau(t)=g^{-2}(t)\left(C_{1}+2 C_{0} \int^{t} g^{2}(s) d s\right)
$$

where $C_{1}$ is a further constant, which finally results in (from (5.61))

$$
m(t)=C_{1} \frac{f(t)}{g^{2}(t)}+C_{0} \int^{t} f(s) d s+2 C_{0} \int^{t} \frac{d}{d t^{\prime}}\left(\frac{f\left(t^{\prime}\right)}{g^{2}\left(t^{\prime}\right)}\right)\left(\int^{t^{\prime}} g^{2}(s) d s\right) d t^{\prime}+C_{2},
$$


in which $C_{2}$ is a constant. There arises three symmetry generators which have in them functions $f(t)$ and $g(t)$. They are

$$
\begin{aligned}
H_{1}= & \frac{\partial}{\partial x} \\
H_{2}= & 2 g^{-2}(t) \int^{t} g^{2}(s) d s \frac{\partial}{\partial t}+\left(x+\int^{t} f(s) d s\right. \\
& \left.\quad+2 \int^{t} \frac{d}{d t^{\prime}}\left(\frac{f\left(t^{\prime}\right)}{g^{2}\left(t^{\prime}\right)}\right)\left(\int^{t^{\prime}} g^{2}(s) d s\right) d t^{\prime}\right) \frac{\partial}{\partial x} \\
H_{3}= & g^{-2}(t) \frac{\partial}{\partial t}+\frac{f(t)}{g^{2}(t)} \frac{\partial}{\partial x} .
\end{aligned}
$$

The commutators are

$$
\left[H_{1}, H_{2}\right]=H_{1},\left[H_{1}, H_{3}\right]=0,\left[H_{2}, H_{3}\right]=-2 H_{3} .
$$

Here the generators span a three-dimensional Lie algebra.

\subsection{Conclusion}

The methodology of [5] was incomplete: the Itô formula was precluded in the temporal Lie point transformation analysis which we have remedied. Overlooking this formula gave rise to transformations that did not provide form invariance. We re-considered two examples from [5] to demonstrate this.

We also note that as a consequence of the equations

$$
Y(\xi)=H(G)+\frac{1}{2} G \Gamma(\tau)
$$

and

$$
Y(\tau)=0
$$

for first order SODEs with one underlying Wiener process, the temporal infinitesimal must at most be a linear function of time. This ensures that the condition

$$
\Gamma\left(e^{\epsilon H}(t)\right)=e^{\epsilon \Gamma(\tau)},
$$

which we derived in Fredericks and Mahomed [2] will always be satisfied. It is this condition that enables us to reconstruct the finite transformations from the infinitesimal ones, which maintain form invariance in each of the two examples from [5]. The Itô formula is an important component of the temporal Lie point symmetry transformation analysis. Without it, reconstructing form invariant finite transformations from the infinitesimal ones is impossible.

Our third example demonstrates that the symmetry generators found still form a Lie algebra even though the condition (5.69) is not satisfied. However, it is not guaranteed that the finite transformations that are recoverable from the infinitesimal ones will guarantee form invariance. 


\section{Acknowledgement}

Ebrahim Fredericks would like to thank the NRF of South Africa for supporting his studies at the University of the Witwatersrand via the grant number SF H2005082200036.

\section{References}

[1] Brzeźniak Zdzisław and Zastawniak Tomasz, Basic Stochastic Processes, Springer, 2002.

[2] Fredericks E and Mahomed F M, Symmetries of First-Order Stochastic Ordinary Differential Equations Revisited, Mathematical Methods in the Applied Sciences 30 (2007), 2013-2025.

[3] Freiduin M, Functional Integration and Partial Differential Equations, Princeton University Press, 1985.

[4] Gaeta G and Quintero N R, Lie-point symmetries and stochastic differential equations, Journal of Physics A: Mathematical and General 32 (1999), 8485-8505.

[5] Meleshko S V, Srihirun B S and Schultz E, On the definition of an admitted Lie group for stochastic differential equations, Communications in Nonlinear Science and Numerical Simulation 12 (8) (2006), 1379-1389.

[6] ØKsendal Bernt, When is a Stochastic Integral a Time Change of a Diffusion?, Journal of Theoretical Probability 3 (2) (1990), 207-226.

[7] ØKsendal Bernt, Stochastic Differential Equations, Springer-Verlag, 1998.

[8] Revuz D and Yor M, Continuous Maringales and Brownian Motion, Springer, 2005.

[9] Ünal G, Symmetries of Itô and Stratonovich Dynamical Systems, Nonlinear Dynamics 32 (2003), 417-426.

[10] Wafo Soh C and Mahomed F M, Integration of stochastic ordinary differential equations from a symmetry standpoint, Journal of Physics A: Mathematical and General 34 (2001), $177-194$. 